\title{
Plan curricular y la formación por competencias de estudiantes de decimo ciclo de Administración-Universidad Nacional Mayor de San Marcos
}

\section{Curricular plan and training by competences of students of the tenth Administration cycle- Mayor de San marcos National University}

\author{
Lic. Jorge Luis Alarcón Cerna
}

\begin{abstract}
RESUMEN
Objetivo: Establecer una relación entre el Plan Curricular y la Formación por Competencias de estudiantes del Décimo Ciclo de la Escuela Profesional de Administración de la Universidad Nacional Mayor de San Marcos, durante el año 2017-2018. Material y métodos: La presente investigación está tipificada como básica, primaria, descriptiva, correlacional, cuantitativa, bivariable y transaccional. La muestra que se obtuvo es de 80 estudiantes, utilizando un software estadístico. Resultados: Existe una relación moderada entre el plan curricular y la formación por competencias. Conclusión: Mostró una relación entre un plan curricular logrado en la formación universitaria de los estudiantes de décimo ciclo que están por egresar de la Escuela Profesional de Administración, Universidad Nacional Mayor de San Marcos.
\end{abstract}

Palabras clave: Currículo, plan curricular, competencias, enseñanza.

\section{ABSTRACT}

Objective: To establish a relationship between the Curriculum Plan and the Training by Skills of students of the Tenth Cycle of the Professional School of Administration of the National University of San Marcos, during 2017-2018. Material and methods: The present investigation is typified as basic, primary, descriptive, correlational, quantitative, bivariable and transactional. The sample obtained is 80 students, using statistical software. Results: There is a moderate relationship between the curricular plan and skills training. Conclusion: It showed a relationship between a curricular plan achieved in the university education of the tenth cycle students who are about to graduate from the Professional School of Administration, Mayor de san Marcos National University.

Keywords: curriculum, curricular plan, competences, teaching

\section{INTRODUCCIÓN}

La presente investigación responde a la necesidad de cómo plantear un currículo para un programa universitario. Si bien cada institución educativa tiene diferentes necesidades y características particulares, trataremos el currículo como un instrumento para dirigir el planeamiento de la enseñanzaaprendizaje en la universidad.

Mejía (2015), indica que existe una correlación significativa entre la variable plan curricular (el currículo en acción) y el desempeño académico por competencias. El autor manifiesta que se puede realizar una retroalimentación que puede modificar el currículo por motivo de las exigencias del mercado laboral tan dinámico y tan cambiante como el nuestro.

Manrique (2009), nos describe el proceso de evaluación del currículo, con el fin de mejorarlo y esto genera cambio en los planes de estudio, el autor propone la creación de comisiones integradas por autoridades, docentes, alumnos y egresados, para ver de qué manera el currículo puede ser adaptado a las competencias exigidas por el mercado laboral. Alejos y Sánchez (2015) plantean que existe una correlación mutua y viceversa entre las variables plan curricular y perfil profesional, ademas proponen un diagnóstico situacional del área de acción del profesional egresado. Lovatón (2011), concluye que la herramienta diagnóstico situacional sirve para saber en qué situación se encuentra la formación de profesionales, a partir del diagnóstico se plantea el currículo. Todos los autores concluyen que el currículo es un documento de guía que las entidades educativas usan para formar a los estudiantes según el perfil profesional de egreso que la entidad propone.

Según Isbell (2014) afirma que el conocimiento de la calidad y duración del programa habría sido fundamental para interpretar los hallazgos longitudinales, la duración más larga de los programas, tiene una relación más fuerte con las competencias generadas por el participante.

Por último, Bycroft (2011), indica que las competencias se pueden dividir en las siguientes cuatro áreas: competencias personales, competencias de conocimiento y habilidad, competencias situacionales y organizacionales y competencias de metodología

Según el modelo de Ayes (2003), las bases teóricas del plan curricular contemplan las dimensiones del currículo: académica (fundamentos y enfoques), laboral (ejecución y principios y metodológica (modelo y diseño).

Las bases teóricas de la formación por competencias, según Larraín y González (2007), son clasificadas de distinta manera por diversos autores. Las que concitan el mayor grado de concenso con competencias básicas o instrumentales, competencias genéricas o transversales o intermadias o generativas o generales y las competencias específicas o técnicas o especificadas.

\section{MATERIALES Y MÉTODOS}

En la presente investigación los instrumentos utilizados fueron como cuestionarios, los cuales se validaron y tienen una alta confiabilidad. Estas herramientas se usaron para recabar información. La técnica utilizada es la encuesta, la cual en casi la totalidad de las preguntas de los instrumentos poseen una escala de valoración (Likert). La población del décimo ciclo es de 222 estudiantes de Administración, de los que se tomó una muestra de 80 estudiantes.

En el análisis estadístico se utilizó el software SPSS 23, para análisis de datos que como resultado nos muestra gráficos y tablas. Para la contrastación de la hipótesis se utilizó el coeficiente de correlación de Spearman, para variables no paramétricas.

La validez y confiabilidad de los instrumentos se realizó de la siguiente manera:

a) Validez de los instrumentos: Mediante juicio de expertos

b) Confiabilidad: Mediante la prueba de Alfa de Cronbach. 


\section{Tabla 1}

Análisis de confiabilidad del plan curricular

\begin{tabular}{lccc}
\hline & $\mathrm{N}$ & Items & Alfa de Crombach \\
\hline $\begin{array}{l}\text { Plan } \\
\text { curricular }\end{array}$ & 45 & 58 & 0,788 \\
\hline
\end{tabular}

Se observa dentro del análisis de consistencia interna «Alfa de Cronbach» del instrumento plan Curricular, existe un nivel de confiabilidad aceptable $(\alpha=0,788)$ en cada uno de los instrumentos.

Tabla 2

Análisis de confiabilidad de la formación por competencias

\begin{tabular}{lccc}
\hline & $\mathrm{N}$ & Items & Alfa de Crombach \\
\hline $\begin{array}{l}\text { Desarrollo de } \\
\text { competencias }\end{array}$ & 45 & 26 & 0,876 \\
\hline
\end{tabular}

Se encontró dentro del análisis de consistencia interna «Alfa de Cronbach» del instrumento formación por competencias, existe un buen nivel de confiabilidad aceptable $(\alpha=0,876)$. Por ello, se realizó una medición objetiva de la característica de interés en el presente estudio.

\section{RESULTADOS}

El $68,8 \%$ de los estudiantes presentan un nivel medio respecto a la percepción al plan curricular y el 31,3\% presenta un nivel bajo respecto al plan curricular.

Tabla 3

Valoración del plan curricular

\begin{tabular}{lcc}
\hline & Frecuencia & Porcentaje \\
\hline Bajo & 25 & 31,3 \\
Medio & 55 & 68,8 \\
Alto & 0 & 0,0 \\
\hline Total & 80 & 100,0
\end{tabular}

El $91,3 \%$ de los estudiantes presentan un nivel medio respecto a la percepción del aspecto académico del plan curricular, el $5 \%$ presenta un nivel bajo y el $3,8 \%$ presenta un nivel alto respecto del aspecto académico del plan curricular.

Tabla 4

Valoración de dimensión de aspectos académicos

\begin{tabular}{lcc}
\hline & Frecuencia & Porcentaje \\
\hline Bajo & 4 & 5,0 \\
Medio & 73 & 91,3 \\
Alto & 3 & 3,8 \\
\hline Total & 80 & 100,0
\end{tabular}

EI 56,3\% presenta un nivel bajo respecto a la formación laboral del plan curricular, el $42,5 \%$ de los estudiantes presentan un nivel medio respecto a la percepción de la formación laboral del plan curricular y el $1,3 \%$ presenta un nivel alto respecto a la formación laboral del plan curricular.

Tabla 5

Valoración de dimensión de formación laboral

\begin{tabular}{lcc}
\hline & Frecuencia & Porcentaje \\
\hline Bajo & 45 & 56,3 \\
Medio & 34 & 42,5 \\
Alto & 31 & 1,3 \\
\hline Total & 80 & 100,0
\end{tabular}

El $87,5 \%$ de los estudiantes presentan un nivel bajo respecto a los aspectos metodológicos al plan curricular y el $12,5 \%$ presenta un nivel medio respecto a los aspectos metodológicos del plan curricular.

Tabla 6

Valoración de dimensión de aspectos metodológicos

\begin{tabular}{lcc}
\hline & Frecuencia & Porcentaje \\
\hline Bajo & 70 & 87,5 \\
Medio & 10 & 12,5 \\
Alto & 0 & 0,0 \\
\hline Total & 80 & 100,0
\end{tabular}

En la tabla 7 se observa que el $71,3 \%$ de los estudiantes percibe un nivel bajo respecto a la formación por competencias y el $28.8 \%$ un nivel medio respecto a la formación por competencias.

Tabla 7

Valoración de formación por competencia

\begin{tabular}{lcc}
\hline & Frecuencia & Porcentaje \\
\hline Bajo & 57 & 71,3 \\
Medio & 23 & 28,8 \\
Alto & 0 & 0,0 \\
\hline Total & 80 & 100,0 \\
\hline
\end{tabular}

En la tabla 8 se observa que el $55 \%$ de los estudiantes perciben un nivel bajo respecto a la formación por competencias básicas y el $45 \%$ un nivel medio respecto a la formación por competencias básicas

Tabla 8

Valoración de dimensión y formación por competencias básicas

\begin{tabular}{lcc}
\hline & Frecuencia & Porcentaje \\
\hline Bajo & 44 & 55,0 \\
Medio & 36 & 45,0 \\
Alto & 0 & 0,0 \\
\hline Total & 80 & 100,0 \\
\hline
\end{tabular}


En la tabla 9 se observa que el $57,5 \%$ de los estudiantes perciben un nivel bajo respecto a la formación por competencias genéricas y el $42,5 \%$ un nivel medio respecto a la formación por competencias genéricas.

Tabla 9

Valoración de dimensión de formación por competencias genéricas

\begin{tabular}{lcc}
\hline & Frecuencia & Porcentaje \\
\hline Bajo & 46 & 57,5 \\
Medio & 34 & 42,7 \\
Alto & 0 & 0,0 \\
\hline Total & 80 & 100,0
\end{tabular}

En la tabla 10 se observa que el $87,5 \%$ de los estudiantes percibe un nivel bajo respecto a la formación por competencias específicas y el $12,5 \%$ un nivel medio respecto a la formación por competencias específicas.

Tabla 10

Dimensión de formación por competencias especificas

\begin{tabular}{lcc}
\hline & Frecuencia & Porcentaje \\
\hline Bajo & 70 & 87,5 \\
Medio & 10 & 12,5 \\
Alto & 0 & 0,0 \\
\hline Total & 80 & 100,0
\end{tabular}

Hipótesis 1

H1a: Existe relación significativa entre los aspectos académicos del plan curricular con la formación por competencias básicas de los estudiantes del décimo ciclo de la Escuela Profesional de Administración, UNMSM-2018.

H1a0: No existe relación significativa entre los aspectos académicos del plan curricular con la formación por competencias básicas de los estudiantes del décimo ciclo de la Escuela Profesional de Administración, UNMSM-2018.

\section{Contrastación de Hipótesis 1}

\section{Tabla 11}

Correlación entre los aspectos académicos y las competencias básicas

\begin{tabular}{lcc}
\hline Estadistica & $\begin{array}{c}\text { Competencias } \\
\text { básicas }\end{array}$ \\
\hline & $\begin{array}{c}\text { Coeficiente de } \\
\text { correlación } \\
\text { Spearman }\end{array}$ & 0,390 \\
Aspectos & Sig. (bilateral) & 0,000 \\
académicos & $\mathrm{N}$ & 80 \\
\hline
\end{tabular}

\section{Hipótesis 2}

H2a: Existe relación significativa entre la formación laboral del plan curricular con la formación por competencias básicas de los estudiantes del décimo ciclo de la Escuela Profesional de Administración, UNMSM-2018.

H2a0: No existe relación significativa entre la formación laboral del plan curricular con la formación por competencias básicas de los estudiantes del décimo ciclo de la Escuela Profesional de
Administración, UNMSM-2018.

Contrastación de Hipótesis 2

Tabla 12

Correlación entre la formación laboral y las competencias básicas

\begin{tabular}{lcc}
\hline Estadistica & $\begin{array}{c}\text { Competencias } \\
\text { básicas }\end{array}$ \\
\hline & $\begin{array}{c}\text { Coeficiente de } \\
\text { correlación } \\
\text { Spearman }\end{array}$ & 0,158 \\
$\begin{array}{l}\text { Formación } \\
\text { laboral }\end{array}$ & Sig. (bilateral) & 0,162 \\
& $\mathrm{~N}$ & 80 \\
\hline
\end{tabular}

Hipótesis 3

H3a: Existe relación significativa entre los aspectos metodológicos del plan curricular con la formación por competencias básicas de los estudiantes del décimo ciclo de la Escuela Profesional de Administración, UNMSM-2018.

H3a0: No existe relación significativa entre los aspectos metodológicos del plan curricular con la formación por competencias básicas de los estudiantes del décimo ciclo de la Escuela Profesional de Administración, UNMSM-2018.

\section{Contrastación Hipótesis 3}

Tabla 13

Correlación entre los aspectos metodológicos y las competencias básicas

\begin{tabular}{lcc}
\hline & Estadistica & $\begin{array}{c}\text { Competencias } \\
\text { básicas }\end{array}$ \\
\hline & $\begin{array}{c}\text { Coeficiente de } \\
\text { correlación } \\
\text { Spearman }\end{array}$ & 0,256 \\
Aspectos & Sig. (bilateral) & 0,022 \\
metodológicos & $\mathrm{N}$ & 80 \\
\hline
\end{tabular}

\section{Hipótesis 4}

H4a: Existe relación significativa entre los aspectos académicos del plan curricular con la formación por competencias genéricas de los estudiantes del décimo ciclo de la Escuela Profesional de Administración, UNMSM-2018.

H4a0: No existe relación significativa entre los aspectos académicos del plan curricular con la formación por competencias genéricas de los estudiantes del décimo ciclo de la Escuela Profesional de Administración, UNMSM-2018

\section{Contrastación de Hipótesis 4}

Tabla 14

Correlación entre los aspectos académicos y las competencias genéricas

\begin{tabular}{lcc}
\hline & Estadistica & $\begin{array}{c}\text { Competencias } \\
\text { básicas }\end{array}$ \\
\hline & $\begin{array}{c}\text { Coeficiente de } \\
\text { correlación Spearman }\end{array}$ & 0,309 \\
Aspectos & Sig. (bilateral) & 0,005 \\
académicos & $\mathrm{N}$ & 80 \\
\hline
\end{tabular}




\section{Hipótesis 5}

H5a: Existe relación significativa entre la formación laboral del plan curricular con la formación por competencias genéricas de los estudiantes del décimo ciclo de la Escuela Profesional de Administración, UNMSM-2018.

H5a0: No existe relación significativa entre la formación laboral del plan curricular con la formación por competencias genéricas de los estudiantes del décimo ciclo de la Escuela Profesional de Administración, UNMSM-2018.

Contrastación de Hipótesis 5

\section{Tabla 15}

Correlación entre la formación laboral y las competencias genéricas

\begin{tabular}{lcc}
\hline & Estadistica & $\begin{array}{c}\text { Competencias } \\
\text { básicas }\end{array}$ \\
\hline & $\begin{array}{c}\text { Coeficiente de } \\
\text { correlación Spearman }\end{array}$ & 0,007 \\
$\begin{array}{l}\text { Formación } \\
\text { laboral }\end{array}$ & Sig. (bilateral) & 0,954 \\
& $\mathrm{~N}$ & 80 \\
\hline
\end{tabular}

\section{Hipótesis 6}

H6a: Existe relación significativa entre los aspectos metodológicos del plan curricular con la formación por competencias genéricas de los estudiantes del décimo ciclo de la Escuela Profesional de Administración, UNMSM-2018.

H6a0: No existe relación significativa entre los aspectos metodológicos del plan curricular con la formación por competencias genéricas de los estudiantes del décimo ciclo de la Escuela Profesional de Administración, UNMSM-2018.

\section{Contrastación de Hipótesis 6}

\section{Tabla 16}

Correlación entre los aspectos metodológicos y las competencias genéricas

\begin{tabular}{lcc}
\hline & Estadistica & $\begin{array}{c}\text { Competencias } \\
\text { básicas }\end{array}$ \\
\hline & $\begin{array}{c}\text { Coeficiente de } \\
\text { correlación Spearman }\end{array}$ & 0,036 \\
$\begin{array}{l}\text { Aspectos } \\
\text { académicos }\end{array}$ & Sig. (bilateral) & 0,751 \\
& $\mathrm{~N}$ & 80
\end{tabular}

\section{Hipótesis 7}

H7a: Existe relación significativa entre los aspectos académicos del plan curricular con la formación por competencias específicas de los estudiantes del décimo ciclo de la Escuela Profesional de Administración, UNMSM-2018.

H7a0: No existe relación significativa entre los aspectos académicos del plan curricular con la formación por competencias específicas de los estudiantes del décimo ciclo de la Escuela Profesional de Administración, UNMSM-2018.

Contrastación de Hipótesis 7
Tabla 17

Correlación entre los aspectos académicos y las competencias especificas

\begin{tabular}{lcc}
\hline & Estadistica & $\begin{array}{c}\text { Competencias } \\
\text { básicas }\end{array}$ \\
\hline & $\begin{array}{c}\text { Coeficiente de } \\
\text { correlación Spearman }\end{array}$ & 0,286 \\
$\begin{array}{l}\text { Aspectos } \\
\text { académicos }\end{array}$ & Sig. (bilateral) & 0,010 \\
& $\mathrm{~N}$ & 80 \\
\hline
\end{tabular}

\section{Hipótesis 8}

H8a: Existe relación significativa entre la formación laboral del plan curricular con la formación por competencias específicas de los estudiantes del décimo ciclo de la Escuela Profesional de Administración, UNMSM-2018.

H8a0: No existe relación significativa entre la formación laboral del plan curricular con la formación por competencias específicas de los estudiantes del décimo ciclo de la Escuela Profesional de Administración, UNMSM-2018.

\section{Contrastación de Hipótesis 8}

\section{Tabla 18}

Correlación entre la formación laboral y las competencias especificas

\begin{tabular}{lcc}
\hline & Estadistica & $\begin{array}{c}\text { Competencias } \\
\text { básicas }\end{array}$ \\
\hline & $\begin{array}{c}\text { Coeficiente de } \\
\text { correlación Spearman }\end{array}$ & 0,262 \\
$\begin{array}{l}\text { Formación } \\
\text { laboral }\end{array}$ & Sig. (bilateral) & 0,019 \\
& $\mathrm{~N}$ & 80 \\
\hline
\end{tabular}

Hipótesis 9

H9a: Existe relación significativa entre los aspectos metodológicos del plan curricular con la formación por competencias específicas de los estudiantes del décimo ciclo de la Escuela Profesional de Administración, UNMSM-2018.

H9a0: No existe relación significativa entre los aspectos metodológicos del plan curricular con la formación por competencia de la Escuela Profesional de Administración, UNMSM-2018.

\section{Contrastación de Hipótesis 9}

\section{Tabla 19}

Correlación entre los aspectos metodológicos y las competencias especificas

\begin{tabular}{lcc}
\hline & Estadistica & $\begin{array}{c}\text { Competencias } \\
\text { básicas }\end{array}$ \\
\hline & $\begin{array}{c}\text { Coeficiente de } \\
\text { correlación Spearman }\end{array}$ & 0,115 \\
Aspectos & Sig. (bilateral) & 0,308 \\
académicos & $\mathrm{N}$ & 80 \\
\hline
\end{tabular}

\section{Hipótesis general}

HG: El plan curricular se relaciona con la formación por competencias de los estudiantes de décimo ciclo de la Escuela Profesional de Administración, UNMSM-2018.

HG0: El plan curricular no se relaciona con la formación por competencias de los estudiantes de décimo ciclo de la Escuela Profesional de Administración, UNMSM-2018. 


\section{Contrastación de general}

«El plan curricular se relaciona con la formación por competencias de los estudiantes de décimo ciclo de la Escuela Profesional de Administración, UNMSM-2018».

Dado que el nivel de significancia es 0.000 menor que 0,05 , se rechaza la hipótesis nula. Existe una correlación significativa y directa en un nivel regular $(r=0,419)$ entre el plan curricular y la formación por competencias al $95 \%$ de confianza.

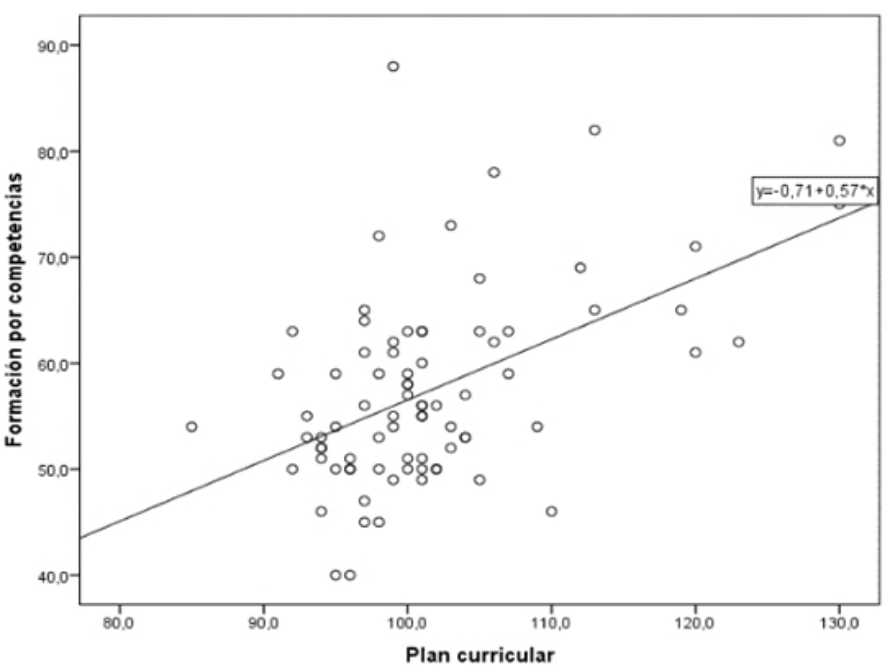

Figura 01 Diagrama de dispersión entre el plan curricular y la formación por competencias

El diagrama de dispersión entre el plan curricular y la formación por competencias explica la presencia de una ligera tendencia lineal, asociación y/o correlación directa.

Tabla 20

Correlación entre el plan curricular y la formación por competencias

\begin{tabular}{lcc}
\hline & Estadistica & $\begin{array}{c}\text { Competencias } \\
\text { básicas }\end{array}$ \\
\hline & $\begin{array}{c}\text { Coeficiente de } \\
\text { correlación Spearman }\end{array}$ & 0,419 \\
Aspectos & Sig. (bilateral) & 0,000 \\
académicos & $\mathrm{N}$ & 80 \\
\hline
\end{tabular}

\section{DISCUSIÓN}

Existe una valoración media acerca de la percepción del alumnado con respecto al plan curricular. Es decir, los alumnos $(68 \%)$ piensan que, de alguna manera, el plan curricular ha influenciado en su formación.

Los alumnos mayoritariamente $(91,3 \%)$ rescatan que los aspectos académicos del plan curricular cumplen medianamente sus objetivos.

Existe una baja valoración con respecto a la formación laboral. La mayoría de alumnos $(56,3 \%)$ piensa que su formación laboral ha sido pobre.

Los alumnos en su mayoría $(87,5 \%)$ cree que en su carrera no se ha desarrollado mucho el aspecto metodológico.

Existe una valoración baja acerca de la percepción del alumnado con respecto a la formación por competencias. Es decir, los alumnos $(71,3 \%)$ piensan que en alguna forma no se han formado competentemente. Solo un $28,8 \%$ de alumnado piensa que medianamente se ha formado competentemente.
Los alumnos mayoritariamente (55\%) piensan que su formación por competencias básicas ha sido pobre, seguido de un $45 \%$ que piensa que su formación por competencias básicas tiene una valoración media.

Los alumnos mayoritariamente $(57,5 \%)$ consideran que su formación por competencias genéricas ha sido pobre, seguido de un $42,5 \%$ que piensa que su formación por competencias genéricas tiene una valoración media.

Los alumnos en general $(87,5 \%)$ perciben que no han recibido o han recibido una baja formación por competencias específicas. Solo un $12,5 \%$ afirma que ha recibido una valoración media en formación por competencias específicas.

Se ha utilizado el coeficiente de Spearman como estadístico no paramétrico debido a que los datos no siguen una distribución normal.

\section{CONCLUSIONES}

Existe una correlación significativa y directa en un nivel regular $(t=0,419)$ entre el plan curricular y la formación por competencia en los estudiantes del décimo ciclo de administración de la Universidad Nacional Mayor de San Marcos.

\section{AGRADECIMIENTOS}

Agradezco a la revista BIG BANG FAUSTINIANO, por permitirme concursar para la publicación de mi artículo de investigación.

\section{REFERENCIAS BIBLIOGRAFICAS}

Alejos, R. B. G y Sánchez H.J. M. I (2015), Tesis de Maestría: Plan curricular y su relación con el perfil profesional de la carrera de Biología de la Universidad Ricardo Palma, Lima-Perú.

Ayes, G. (2003). El Diseño Curricular. Informe final de Proyecto de Investigación. ISPETP, Ciudad de La Habana, Cuba.

Bycroft T. (2011), Tesis Doctoral: Percepciones de expertos en investigación con respecto a las competencias necesarias para practicar una investigación segura con asuntos humanos, Universidad Estatal de Oklahoma, EEUU.

Isbell A. M. (2014), Tesis Doctoral: Análisis de las relaciones entre programas / actividades extracurriculares y competencias, Universidad de Claremont, EEUU.

Larraín, U., A. M. y González F., L. E. (2007), Formación universitaria por competencias, Chile.

Lovatón S.P.F. (2011), Tesis de Maestría: El Diagnóstico Situacional: herramienta indispensable en la elaboración del currículo para la formación académico-profesional del comunicador social al 2011, Lima-Perú.

Manrique V. L. (2009), Tesis de Maestría: La evaluación procesual del currículo y su efecto en el plan de estudios de una carrera de pregrado de la PUCP. Estudio de caso. Lima-Perú.

Mejía, M. R. (2015) “El currículo en el siglo XXI”, Lima-Perú. Editorial: Escuela Abierta. p 4. 